\title{
Efecto de la transpiración sobre la escorrentía en dos tipos de bosque de la cordillera de la costa, sur de Chile
}

\section{(Transpiration effect on runoff in two types of forest of the coast mountains, southern Chile)}

\author{
Gina Berrones ${ }^{1}$, Carlos Oyarzún²
}

\begin{abstract}
Resumen:
En esta investigación se comparó la tasa de transpiración y su efecto en la escorrentía en dos microcuencas con bosque nativo siempreverde (Bonifacio) y deciduo (Las Palmas) durante el cambio estacional primavera-verano. Las variables utilizadas fueron precipitación, escorrentía, índice de área foliar, transpiración de especies, y distribución del agua en el suelo. Los resultados mostraron un mayor consumo de agua en el bosque deciduo traduciéndose en una mayor evapotranspiración con 2273,6 mm en comparación al bosque siempreverde con 1740 $\mathrm{mm}$, generando una mayor escorrentía en este último. La comparación entre los índices de área foliar de los dos bosques mostraron diferencia, sobretodo en la primavera para el bosque deciduo, ya que al aumentar su tasa transpiratoria por incremento de biomasa, generó una menor escorrentía a pesar que existieron mayores precipitaciones. Además las especies del bosque siempreverde consumieron el agua de los primeros centímetros (10-20) del perfil del suelo a diferencia del bosque deciduo, donde se observó una disminución en la humedad del suelo a una profundidad un poco mayor $(40 \mathrm{~cm})$. En cuanto al rendimiento hídrico, se pudo mostrar con este trabajo que el bosque siempreverde es más eficiente que el bosque deciduo durante el período de activo crecimiento primavera-verano.
\end{abstract}

Palabras clave: Balance hídrico; bosque nativo siempre verde; bosque nativo deciduo; transpiración; escorrentía.

\begin{abstract}
:
The present work compares the rate of transpiration and its effect in the runoff of watershed covered with evergreen (Bonifacio) and deciduous (Las Palmas) native forests, during the spring-summer season change. We used as variables the precipitation, runoff, leaf area index, transpiration of the species, and the distribution of water in the soil. The results show a greater consumption of water in the deciduous forest, by a greater evotranspiration of $2273.6 \mathrm{~mm}$ compared to the evergreen forest of $1740 \mathrm{~mm}$, which generates a greater runoff than the last one. The comparison between leaf area indexes of the two forests shows a significant difference, especially during spring for the deciduous forest, since the increase of the transpiration rate due to the increase of biomass, generates fewer runoffs even though in this location there were more precipitations. Moreover, the species of the evergreen forest consumed the water of the first centimeters (10-20) of the soil profile; in contrast, in the deciduous forest we observed a greater decrease in the deep soil water content $(40 \mathrm{~cm})$. Regarding water yield, we show that the evergreen forest is more efficient than deciduous forest, during the period spring-summer.
\end{abstract}

Keywords: Water balance; native evergreen forests; native deciduous forests; transpiration; runoff.

\footnotetext{
${ }^{1}$ Universidad Tecnológica Equinoccial, Facultad de Ciencias de la Ingeniería, Quito - Ecuador (bggm98383@ute.edu.ec)

2Universidad Austral de Chile, Instituto de Ciencias de la Tierra y Evolución, Valdivia - Chile (coyarzun@uach.cl)
} 


\section{Introducción}

Dentro de un ecosistema forestal, la evaluación del balance hídrico es importante para conocer la cantidad de agua que demanda la vegetación para su consumo. Las condiciones climáticas tienen una relación directa en el balance hídrico, donde los impactos hidrológicos de los bosques dependen, entre otros, de las condiciones climatológicas del lugar (Oyarzún, Nahuelhual, y Núñez, 2006). Una diferencia en el consumo de agua sólo se pondrá de manifiesto si el clima tiene períodos de excedentes hídricos, permitiendo que las reservas de agua del suelo se repongan. (Vazken, 2003).

El ciclo hidrológico implica un complejo despliegue de movimientos y transformaciones del agua (Echeverría, Huber, y Taberlet, 2007) que queda disponible en el suelo dependiendo de las precipitaciones que llegan sobre él y de la capacidad del suelo para almacenarla (Pizarro, Vallejos, Balocchi, Morales, y León, 2005). Debido a que una cubierta vegetal de tipo boscoso está relacionado con la redistribución de las precipitaciones, cualquier tipo de alteración dentro de este ecosistema podría traer consecuencias locales sobre el balance hídrico.

La escorrentía es el agua generada por una cuenca en la forma de flujo superficial, subsuperficial y subterráneo. Se forma cuando las precipitaciones superan la capacidad de infiltración del suelo, cuando los compartimentos del suelo estén saturados de agua.

Una menor evapotranspiración (ET) indica una mayor escorrentía para la precipitación dada. Cuando la precipitación es mayor que la ET potencial, la ET puede variar ampliamente de acuerdo al tipo de vegetación. Los cambios en la escorrentía debido a los cambios de vegetación indican cambios en la ET (Komatsu, Kume, y Otsuki, 2010), éste es un proceso en el cual influyen varios factores, tales como área foliar total y la capacidad transpiratoria de las especies, así como el tipo de suelo, la topografía y las condiciones meteorológicas (Quintana, 2009).

La demanda hídrica no es igual en todos los suelos. La captación de agua del suelo por las plantas está determinada por el control fisiológico de la pérdida de agua a través de una combinación de fluctuaciones en la conductancia estomática y la cantidad de follaje (Roberts, 2000). En el proceso de transpiración, el vapor de agua se difunde a través de las aberturas de los estomas de las hojas, y el proceso de difusión está representada por una resistencia estomática.

La resistencia estomática de la vegetación juega un papel importante en la determinación de cómo la energía disponible se divide en calor sensible y latente, en particular para los bosques cuya resistencia aerodinámica tiende a ser mucho más pequeña que la resistencia del dosel. La conductancia estomática depende de los factores ambientales del entorno como la radiación solar, la temperatura del aire, déficit de saturación del aire, potencial hídrico foliar y la concentración externa de $\mathrm{CO}_{2}$ (Schelde, 1996). 
Entre especies arbóreas difieren los rasgos estructurales y las características fisiológicas, lo cual influye tanto en la dinámica vertical como horizontal del agua del suelo (Krämer y Hölscher 2010). Puesto que existe una interacción entre plantas, particularmente de diferentes especies, estudios relativamente recientes han mostrado los efectos competitivos e interactivos de las plantas en relación al agua y de la necesidad de considerar los controles y resultados del medio ambiente sobre la vegetación y de cómo éstos podrían diferir de las respuestas observadas sobre plantas individuales (Roberts, 2000).

La transpiración de las plantas depende de factores ambientales como temperatura, presión, intensidad de luz, velocidad del viento y suministro de agua a la raíz, así como de las características de cada especie. De esta manera existirán variaciones entre tasas de transpiración de especies siempre verdes y especies deciduas. Estimaciones experimentales realizadas por Huber, Oyarzún, y Oñate (1986) sobre la variación de las tasas de transpiración potencial en algunas especies de bosque nativo chileno (bosque templado lluvioso) como Gevuina avellana (avellano), Drymis winteri (canelo), Embothrium coccineum (notro), Aextoxicon punctatum (olivillo) y Eucryphia cordifolia (ulmo), mostraron valores entre 109 y 267 litros de agua por $\mathrm{m}^{-2}$ de superficie foliar año, y estos rangos están correlacionados positivamente con la densidad de estomas.

Durante los períodos donde disminuye la disponibilidad de agua, en verano por descenso de lluvias, y en invierno por los efectos de las bajas temperaturas, las hojas de los árboles deciduos están parcialmente desarrolladas, mientras que las de los siempreverdes están en su desarrollo normal, por lo tanto, las especies deciduas presentarán tasas de crecimiento marcadamente estacionales y las siempreverdes tasas de crecimiento más estables durante todo el año (Oyarzún y Zúñiga 2009).

La biomasa foliar, medida a través del LAI (leaf area index) (índice de área foliar), determina la cantidad de radiación solar fotosintéticamente activa absorbida, la que se encuentra estrechamente relacionada con la productividad primaria neta de la vegetación (Flores y Allen 2004), siendo este índice un importante parámetro indicador de diversos aspectos funcionales en comunidades vegetales (Barrantes y Gracia 1989). Estudios de Cornish y Vertessy (2001) han demostrado que el LAI tiene un valor explicativo de utilidad como el más fuerte indicador en el decrecimiento de los rendimientos hídricos.

EI LAI varía ampliamente entre tipos y especies de plantas en respuesta al agua disponible y al estado nutricional del suelo (Roberts, 2000).

Con un alto porcentaje de área foliar, existirá mayor absorción de radiación solar y, por tanto, mayor producción de biomasa. La eficiencia con que esa radiación absorbida es convertida a biomasa cambia dependiendo de las condiciones del medioambiente. El LAl presenta una 
dinámica estacional con un máximo durante el verano y de acuerdo al estudio realizado por Whitehead, Kelliher, Frampton, y Godfrey (1994) en Nueva Zelanda, cambios de hasta un 100\% desde invierno a verano son posibles.

Debido a que los bosques siempreverdes de la Cordillera de la Costa de Chile forman un ecosistema único en el mundo, son de gran significancia ecológica por presentar una alta heterogeneidad florística y estructural, resultando un foco de atención, al estar expuestos a cierto deterioro debido al reemplazo de estos bosques por plantaciones exóticas, las cuales constituyen un problema de manejo sustentable, con fuertes efectos sobre el balance hídrico y ciclo de nutrientes (Oyarzun et al. 2006). Los bosques lluviosos siempreverde valdivianos se consideran como el centro de máxima biomasa leñosa y riqueza de especies arbóreas de la región, en parte debido a las diferentes combinaciones de tipos de hábitats producidos por cambios abióticos (Lara, Rutherford, y Montory, 1999).

En el sur de Chile se han realizado diversos estudios sobre los bosques nativos y su interacción con algunos aspectos del balance hídrico como la intercepción (por ej. Huber y Oyarzún 1992; Oyarzún et al. 2011) y el manejo de bosques y rendimiento hídrico (por ej. Lara et al. 2009; Oyarzún et al. 2009). Sin embargo, se desconocen las relaciones que existen entre el crecimiento de las distintas especies arbóreas nativas, sus tasas de transpiración y el rendimiento hídrico. Por lo tanto, en el presente estudio se investigó el efecto de las tasas de transpiración sobre la escorrentía en el período primavera-verano, en bosque siempreverde y deciduo, en dos microcuencas localizadas en la cordillera de la costa, sur de Chile.

Ya que durante la primavera-verano, etapa de mayor crecimiento vegetacional, las especies arbóreas deciduas incrementarán mayormente su área foliar que las del bosque siempreverde, traduciéndose en mayores tasas de transpiración, y durante la etapa de rebrote de especies arbóreas deciduas, se espera un cambio negativo en la escorrentía de la cuenca, que será proporcional al aumento en su área foliar. Este patrón, será menos marcado en la cuenca cubierta de especies siempreverdes, debido a un menor aumento de su área foliar. De esta manera se planteó determinar la transpiración, conductancia estomática y eficiencia hídrica de las principales especies arbóreas (deciduas y siempreverde) en el periodo primavera-verano, determinar los cambios temporales en la escorrentía de los dos tipos de bosques, y correlacionar el rendimiento hídrico en la transición primavera-verano, con la tasa de transpiración y el tipo de vegetación (deciduo y siempreverde) en cada microcuenca.

\section{Materiales y métodos}

\section{1. Área de estudio}


La investigación se realizó en dos microcuencas (Bonifacio y Las Palmas) ubicadas en la Cordillera de la Costa (39 $50^{\prime} S$ y $73^{\circ} 10^{\prime} \mathrm{W}$ ) cerca de la ciudad de Valdivia. Bonifacio, tiene un área de 3,13 hectáreas y presenta una cobertura de bosque nativo siempreverde. Las especies tomadas en cuenta para el estudio y las mediciones respectivas, fueron: Gevuina avellana (avellano), L, philippiana (tepa), A. punctatum (olivillo) y Drimys winteri (canelo), debido a su mayor número de arboles por hectárea y porcentaje de área basal. El suelo se encuentra clasificado como rojo arcilloso con basamento metamórfico. El uso de este suelo estaba marcado por el pastoreo de animales que degradaron paulatinamente el bosque nativo.

Las Palmas, tiene una superficie de 8,1 hectáreas. El bosque deciduo se encuentra conformado principalmente por Nothofagus oblicua (roble) con 400 árboles/ha, un área basal del 96\% del total y además están presentes otras especies, tomándose en cuenta para este estudio además del roble, Chusquea quila (quila), como la más predominante.

Las características físicas de los suelos de Bonifacio y de Las Palmas se describen en la tabla 1. En cada sitio se describió un perfil de suelo a través de una calicata, determinándose la densidad aparente del suelo a distintas profundidades (tercios superior, medio e inferior del perfil) a través del método del cilindro. En Bonifacio se encontró un porcentaje de materia orgánica de 17,8\%, el sustrato geológico es un complejo metamórfico dominado principalmente de esquistos micáceos con lentes de cuarzo intercalados. De acuerdo a los porcentajes tanto de arena, limo y arcilla se define como un suelo rojo- arcilloso derivado de cenizas volcánicas antiguas, depositados sobre el complejo metamórfico. En Las Palmas, el porcentaje de materia orgánica fue de $16,7 \%$ en los primeros centímetros y de 7,5\% en el horizonte inferior, y de acuerdo a la cantidad de arena, limo y arcilla presente en este suelo se lo puede denotar como limo arcilloso.

\subsection{Mediciones de terreno}

El presente estudio se realizó en el periodo invierno - primavera - verano (agosto - marzo), tomando como base fundamental la transición primavera - verano.

Las variables en estudio fueron medidas mediante varios instrumentos (pluviómetro, fluviómetro, sonda PR2, analizador de gas infrarrojo, y analizador LAI), algunos de los cuales ya se encontraban previamente instalados en las microcuencas. Las variables de humedad del suelo, transpiración e índice de área foliar fueron medidos mensualmente, mientras que las variables de precipitación y escorrentía fueron registrados diariamente.

Los valores de precipitación dentro de las cuencas, fueron cuantificados a través de pluviómetros conectados a un datalogger $\mathrm{HOBO}$, los que proporcionaron información acerca del inicio, duración e intensidad de la lluvia, y que estuvieron ubicados en un área abierta sin vegetación $10 \mathrm{~m}$ a la redonda. La cantidad de agua que alcanza la superficie corresponde a la precipitación en el área, menos la intercepción. Esta última se estima midiendo la precipitación directa y el escurrimiento 
fustal. Sin embargo, no se obtuvieron datos de estos últimos por lo que se asume una intercepción igual a cero.

El balance hídrico de las microcuencas se calculó con la ecuación:

$E V T R=P-Q \pm \Delta S$

Donde:

$\mathrm{P}=$ precipitación

$Q=$ escorrentía

EVTR = evapotranspiración

$\Delta S=$ variación del contenido de agua del suelo

Tabla 1. Características físicas del suelo de las microcuencas

\begin{tabular}{|c|c|c|c|c|c|c|c|}
\hline Microcuenca & $\begin{array}{l}\text { Prof. } \\
\text { (cm) }\end{array}$ & $\begin{array}{l}\text { Arena } \\
\text { (\%) }\end{array}$ & $\begin{array}{l}\text { Limo } \\
\text { (\%) }\end{array}$ & $\begin{array}{l}\text { Arcilla } \\
\text { (\%) }\end{array}$ & $\begin{array}{l}\text { M.O } \\
\text { (\%) }\end{array}$ & $\begin{array}{l}\text { D.aparente } \\
\left(\mathrm{gm}^{-3}\right)\end{array}$ & $\begin{array}{l}\text { Porosidad } \\
\text { (\%) }\end{array}$ \\
\hline \multirow[t]{2}{*}{ Bonifacio } & $0-29$ & 26,0 & 32,5 & 41,5 & 17,8 & 0,58 & 80,3 \\
\hline & $29-65$ & & & & & 0,76 & 71,8 \\
\hline \multirow[t]{2}{*}{ Las Palmas } & $0-20$ & 8,0 & 45,7 & 46,3 & 16,7 & 0,66 & 69,1 \\
\hline & $20-40$ & & & & 7,5 & 0,77 & 65,7 \\
\hline
\end{tabular}

Para determinar la escorrentía en cada microcuenca, se contó con la previa instalación de un fluviómetro con $60^{\circ}$ de salida y dotado con registradores Diver que miden la altura del nivel de agua cada 30 minutos, los cuales fueron calibrados con aforos de terreno para determinar el caudal.

La humedad del suelo se midió a través de una sonda PR2, que proporciona un buen rendimiento en todo tipo de suelo, con una mínima influencia de salinidad o temperatura. Se tomó 6 lecturas hasta una profundidad de 1 metro $(10,20,30,40,60$ y $100 \mathrm{~cm})$. Dentro de cada sitio de estudio se tomó 4 puntos de muestreo, dos de los cuales se encontraban cercanos a las raíces de un árbol y los otros dos puntos se encontraban en un sitio donde no influyeran las raíces de alguno de ellos. Todas las lecturas se realizaron con una frecuencia mensual para obtener los datos que permitieran correlacionar la humedad del suelo con la transpiración de las especies. El porcentaje de humedad del suelo fue transformado a mm de volumen de agua, mediante la multiplicación del valor del porcentaje por la profundidad del perfil del suelo en mm y dividido por 100. 
La transpiración de las especies se realizó mediante un analizador de gas infrarrojo (IRGA) "Infrared Gas Analyzer". La medición fue realizada entre las primeras horas de la mañana, medida con temperatura controlada de $20 \pm 2{ }^{\circ} \mathrm{C}$ y con luz en la cual se encontraban los máximos de transpiración, que fueron de 106 y $484 \mu \mathrm{mol}$ de fotones $\mathrm{m}^{-2} \mathrm{~s}^{-1}$ para las especies sombratolerantes e intolerantes, respectivamente. Estos experimentos ya fueron realizados previamente para determinar los $\mu \mathrm{mol}$ fotones a través de una curva de luz. Se realizaron dos repeticiones por cada especie de cada bosque, y el aparato tomó 5 medidas en un lapso de 2 minutos cada una, obteniéndose por cada especie 5 puntos de valoración.

El índice de área foliar (LAI) "Leaf Area Index" fue medido dentro de cada cuenca mediante transectos, con el analizador LAI 2000 (Li-Cor, Lincoln, Nebraska, USA). Este índice se obtuvo a partir de mediciones de la radiación mediante un sensor óptico "ojo de pez", sobre y bajo el dosel que determina la intercepción de luz en cinco ángulos. Se realizaron 3 transectos en cada bosque para medir dicho índice, obteniéndose por tanto 3 repeticiones.

Las variables ambientales luminosidad y temperatura del aire se registraron con un $\mathrm{HOBO}$ Pendant, los que se instalaron en lugar abierto y bajo el dosel de cada bosque.

\subsection{Análisis de los datos}

Para analizar los datos obtenidos en todas las mediciones, se utilizaron los programas Statistica y Sigmaplot y las tendencias gráficas de todas las variables se realizaron en frecuencias mensuales para describir el comportamiento de éstas. Además se realizaron pruebas de normalidad para escoger que tipo de correlación se manejaría (Pearson o Spearman). Los datos resultaron no ser normales, por lo tanto se realizó correlaciones de Spearman en todas las variables, entre los parámetros ambientales y de crecimiento versus la transpiración. Además se añadió la correlación de la evapotranspiración con el índice de área foliar, y de la escorrentía con la transpiración para comparar las respuestas entre los bosques. Dentro del balance hídrico por microcuenca se aplicó un ANOVA de Kruskall Wallis para observar si la diferencia en el índice de escorrentía de ambos bosques era significativa.

\section{Resultados}

\subsection{Transpiración de las especies}

Los resultados de este estudio, muestran diferentes tasas de transpiración para las diversas especies arbóreas en los dos bosques. Para el bosque siempreverde, se observó que durante el mes de octubre, tanto el avellano como el canelo presentaron la mayor tasa de transpiración. En Septiembre, el olivillo presentó su valor más alto transpiratorio y posteriormente en los siguientes meses se mantuvo más o menos estable. Para la tepa se observó un incremento paulatino hasta 
alcanzar en noviembre su punto más alto de transpiración. Entre las especies que conforman este bosque, esta fue la especie con el valor más bajo de transpiración durante el estudio, (Figura 1).

Mientras que para el bosque deciduo, la quila mostró valores más o menos estables durante todo el período de estudio, y solamente en los meses de enero a marzo descendió su tasa transpiratoria. Los resultados obtenidos para el roble mostraron un crecimiento de octubre a diciembre, descendiendo la tasa transpiratoria hasta febrero y en marzo alcanzó su punto más alto transpiratorio, (Figura 1).

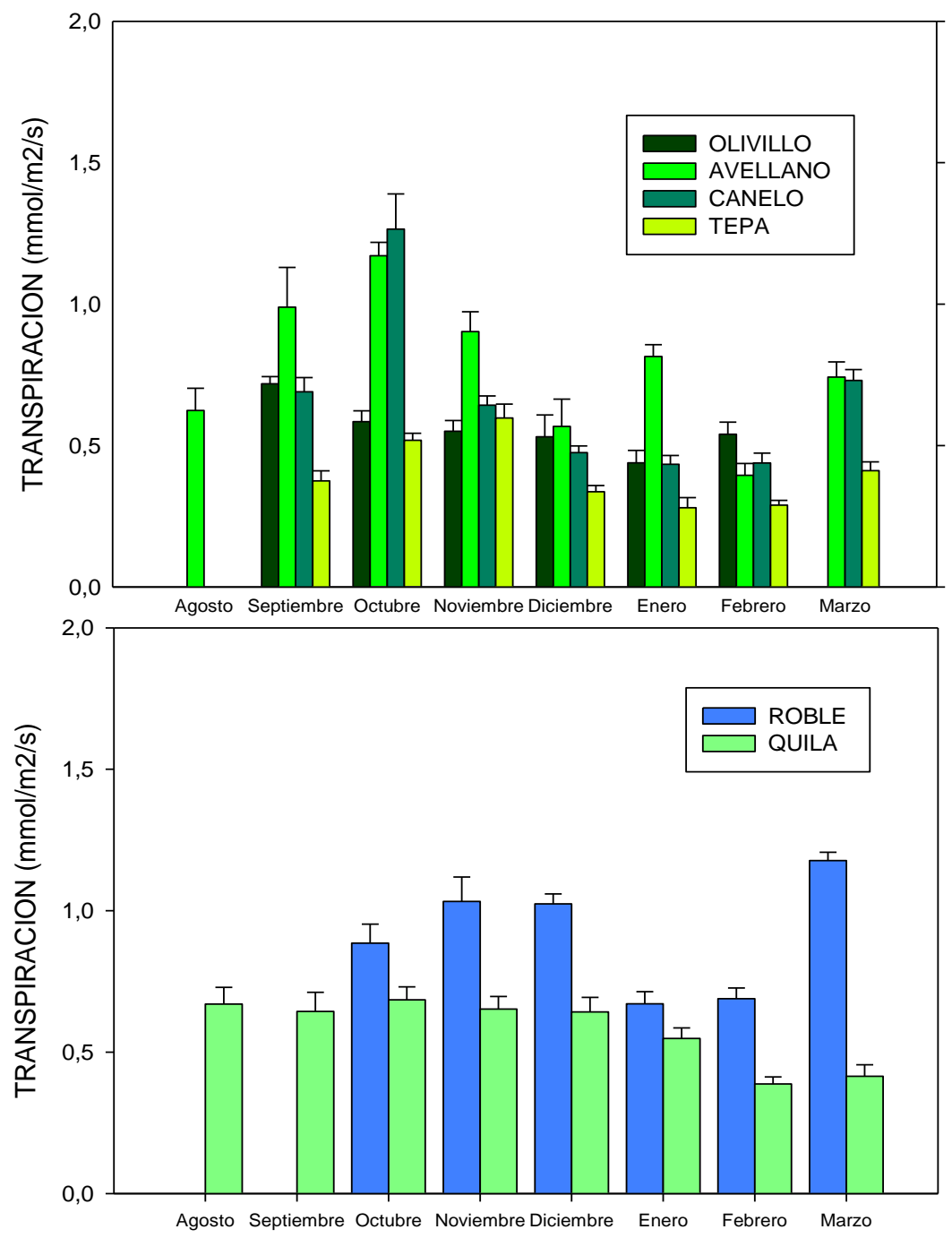


Figura 1. Variaciones mensuales de la transpiración en las principales especies arbóreas de los dos bosques. Superior: Bosque siempreverde, Inferior: Bosque deciduo

La correlación entre la transpiración y la precipitación para el bosque siempreverde no fue significativa, mientras que para el bosque deciduo los resultados mostraron que solamente para el roble existió una correlación significativa negativa $r=-0,151$ y $P=0,039$. (Tabla 2).

La correlación entre la transpiración y la escorrentía del bosque siempreverde mostró significancia para el canelo $r=-0,191 ; P=0,09$ y para la tepa $r=-0,238 ; P=0,001$; mientras que para el bosque deciduo se presentaron resultados significativos para las dos especies, roble $r=-0,515 ; P=0,000$ y quila $r=0,577 ; P=0,000$ (Tabla 2).

Los resultados obtenidos para la correlación entre la transpiración de los bosques y la humedad del suelo no mostraron una diferencia entre las especies a diferentes profundidades. $Y$ solamente a los $60 \mathrm{~cm}$ de profundidad del perfil, la tepa tuvo significancia $r=-0,828 ; P=0,041$ (Tabla 2).

Tabla 2. Correlación Transpiración - Precipitación, Escorrentía, Humedad del suelo, LAl para las microcuencas con bosque nativo siempreverde y bosque nativo deciduo

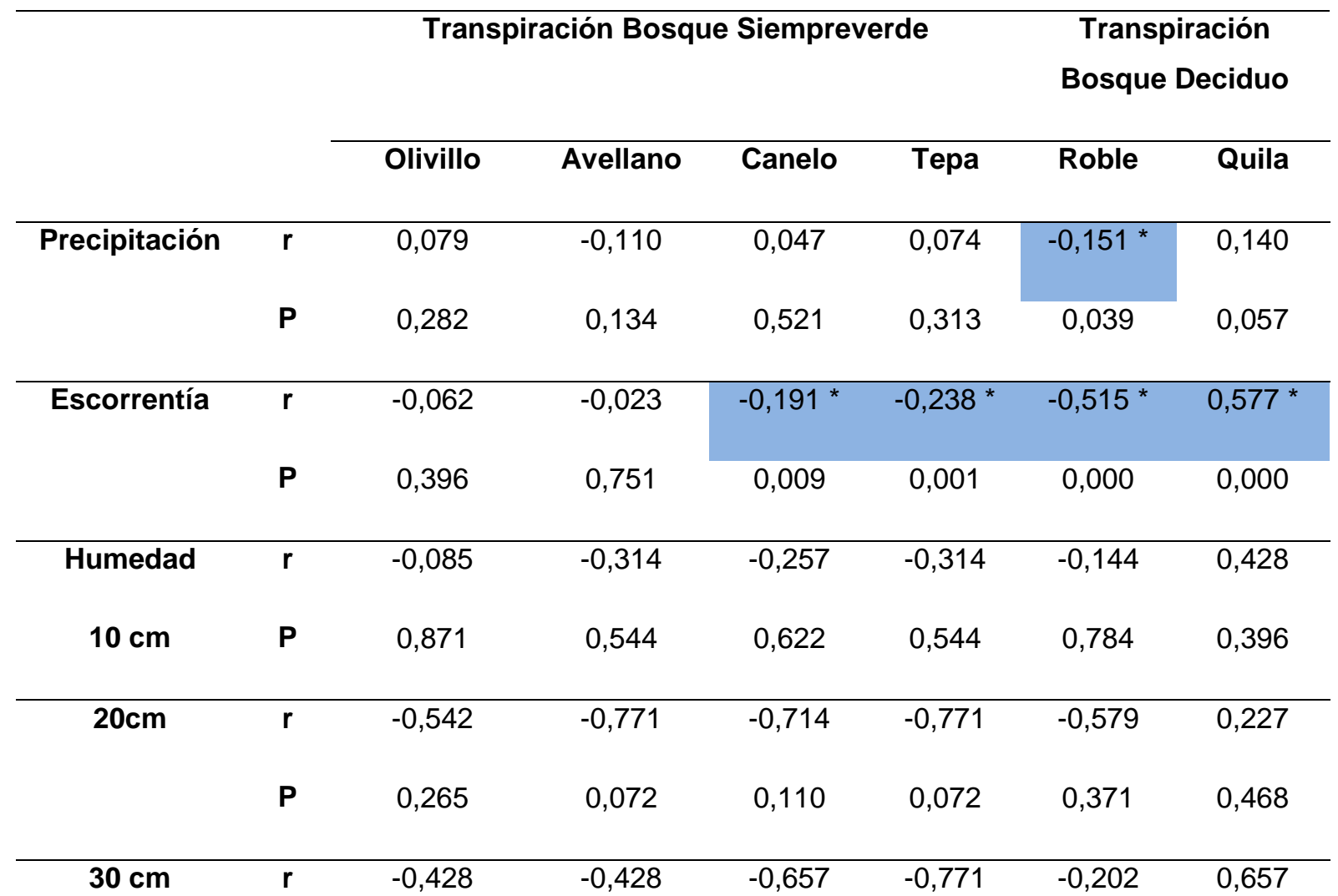




\begin{tabular}{|c|c|c|c|}
\hline 0,396 & 0,396 & 0,156 & 0,072 \\
\hline
\end{tabular}

\begin{tabular}{|c|c|c|c|c|c|c|c|}
\hline \multirow[t]{2}{*}{$40 \mathrm{~cm}$} & $r$ & $-0,142$ & $-0,257$ & $-0,257$ & $-0,600$ & 0,057 & 0,085 \\
\hline & $\mathbf{P}$ & 0,797 & 0,622 & 0,622 & 0,208 & 0,913 & 0,871 \\
\hline \multirow[t]{2}{*}{$60 \mathrm{~cm}$} & $r$ & $-0,485$ & $-0,657$ & $-0,600$ & $-0,828$ * & $-0,318$ & 0,085 \\
\hline & $\mathbf{P}$ & 0,328 & 0,156 & 0,208 & 0,041 & 0,537 & 0,871 \\
\hline \multirow[t]{2}{*}{$100 \mathrm{~cm}$} & $r$ & $-0,085$ & $-0,314$ & $-0,142$ & 0,028 & 0,173 & $-0,600$ \\
\hline & $\mathbf{P}$ & 0,871 & 0,544 & 0,787 & 0,957 & 0,741 & 0,208 \\
\hline \multirow[t]{2}{*}{ LAI } & $r$ & 0,142 & 0,085 & 0,028 & 0,028 & 0,840 * & 0,828 * \\
\hline & $\mathbf{P}$ & 0,787 & 0,871 & 0,957 & 0,957 & 0,036 & 0,041 \\
\hline
\end{tabular}

$\left({ }^{*}\right)$ Las correlaciones marcadas son significativas $p<0,05$

\subsection{Crecimiento de las especies}

La correlación obtenida entre el Índice de área foliar (LAI) y la transpiración de las especies para los dos tipos de bosque, mostró que no existió una correlación significativa para el bosque siempreverde en ninguna de sus especies representativas, mientras que para el bosque deciduo si se obtuvo una correlación positiva para el roble $r=0,840 ; P=0,036$ y para la quila $r=0,828 ; P=$ 0,041. (Tabla 2).

Los resultados obtenidos de la variable índice de área foliar (LAI) mostraron una diferencia en el comportamiento mensual entre los dos bosques. Así en el bosque siempreverde se observó una tendencia a incrementar su biomasa foliar en forma paulatina durante los meses de estudio, mientras que en el bosque deciduo se observó un marcado incremento en la biomasa y fue mayor que en el bosque siempreverde (Figura 2).

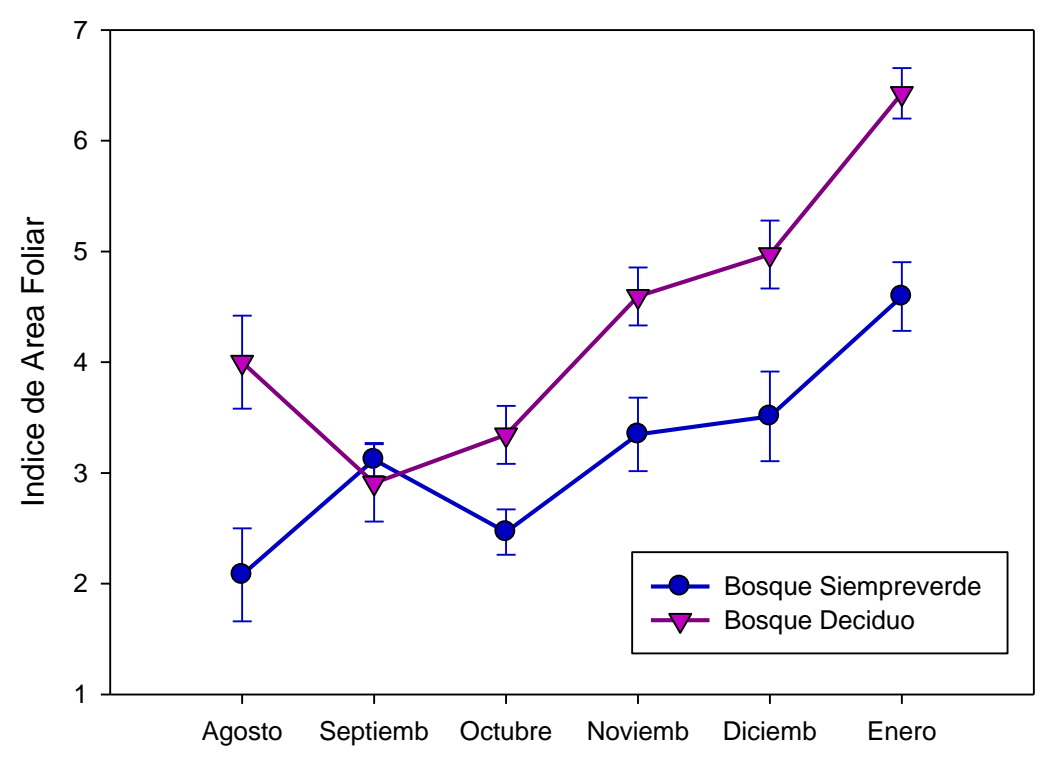


Figura 2. Comportamiento mensual de LAl de los bosques en estudio

\subsection{Comportamiento Hidrológico}

En el balance hídrico mensual de los dos tipos de bosque, la precipitación total fue de 858,3 $\mathrm{mm}$ en el bosque siempreverde y $918 \mathrm{~mm}$ en el bosque deciduo. La escorrentía total en el mismo periodo fue de 342,2 $\mathrm{mm}$ en el bosque siempreverde mientras que en el bosque deciduo fue de $130,4 \mathrm{~mm}$. Se presentó mayor evapotranspiración en el bosque deciduo que en el bosque siempreverde, con valores de 2273,6 y $1740 \mathrm{~mm}$ respectivamente, (Tabla 3).

Se encontraron correlaciones significativas entre la precipitación y la escorrentía, de esta manera para el bosque siempreverde se obtuvo una correlación positiva $r=0,282 ; P<0,01$ y para el bosque deciduo $r=0,386 ; P<0,01$. Las variaciones diarias de la precipitación y escorrentía para los 2 sitios mostraron una clara tendencia directamente proporcional de manera que a mayor precipitación existió mayor escorrentía. En la figura 3, se muestra la variación diaria de la precipitación y la escorrentía en las dos microcuencas del estudio.

Tabla 3. Balance hídrico mensual de los dos tipos de bosque

Bosque Siempreverde (Bonifacio)

\section{MESES}

\begin{tabular}{lrrrrrrrrr}
\hline & Agosto & Septiembre & Octubre & Noviembre & Diciembre & Enero & Febrero & Marzo & Total \\
\hline $\mathbf{P}(\mathbf{m m})$ & 172,7 & 51,1 & 95,9 & 211,6 & 157,1 & 53,1 & 22,3 & 94,2 & 858,3 \\
$\mathbf{Q}(\mathbf{m m})$ & 75,3 & 58,7 & 34,7 & 40,2 & 34,8 & 33,0 & 25,6 & 39,7 & 342,2 \\
$\mathbf{Q} / \mathbf{P}$ & 0,4 & 1,1 & 0,3 & 0,1 & 0,2 & 0,6 & 1,1 & 0,4 & \\
Evtr & 97,3 & 122,9 & 238,9 & 278,5 & 345,3 & 128,3 & 250,4 & 278,2 & 1740,0 \\
$\mathbf{\Delta S}$ & & 130,5 & 177,6 & $-107,1$ & $-223,0$ & 108,2 & 253,7 & $-223,7$ & \\
\hline
\end{tabular}

Bosque Deciduo (Las Palmas)

MESES

Agosto Septiembre Octubre Noviembre Diciembre Enero Febrero Marzo Total 


$\begin{array}{lrrrrrrrrr}\mathbf{P}(\mathbf{m m}) & 396,8 & 85,3 & 89,5 & 99,0 & 82,4 & 52,2 & 23,4 & 89,2 & 918,0 \\ \mathbf{Q}(\mathbf{m m}) & 32,9 & 29,5 & 17,4 & 15,1 & 13,0 & 9,8 & 7,1 & 5,1 & 130,4 \\ \mathbf{Q} / \mathbf{P} & 0,08 & 0,3 & 0,2 & 0,1 & 0,1 & 0,1 & 0,3 & 0,0 & \\ \text { Evtr } & 363,8 & 385,6 & 257,4 & 484,1 & 280,9 & 138,8 & 224,0 & 138,8 & 2273,6 \\ \mathbf{\Delta S} & & 329,8 & 185,3 & -400,2 & 211,5 & 96,5 & 207,7 & 54,7 & \end{array}$

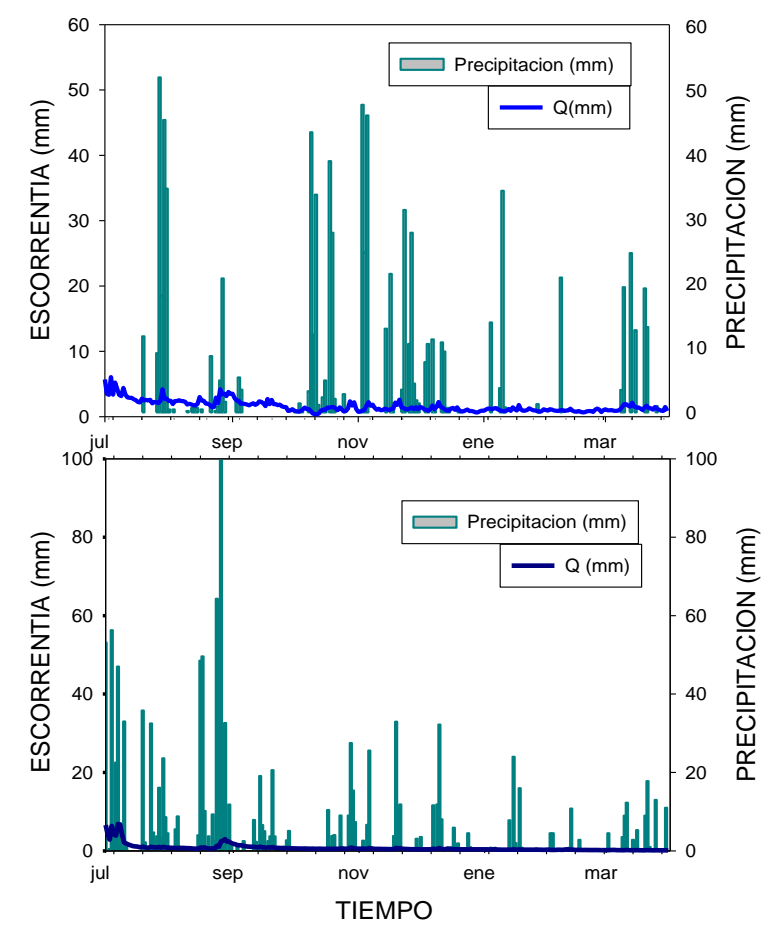

Figura 3. Variaciones diarias de la Precipitación y Escorrentía de las microcuencas. Superior: Bosque siempreverde; Inferior: Bosque deciduo

La humedad del suelo mostró que en el bosque siempreverde, en los primeros $10 \mathrm{~cm}$ de profundidad del perfil la diferencia entre la cantidad de agua disponible fue notable entre el invierno y el verano, siendo mayor en invierno. En los $20 \mathrm{~cm}$, la humedad del suelo disminuyó sin diferencia de estación y a partir de los $40 \mathrm{~cm}$ de profundidad, hubo mucha mayor disponibilidad de agua. Esto permite inferir que las plantas están tomando el agua de los primeros $\mathrm{cm}$ de humedad del suelo solamente. Mientras que para el bosque deciduo se observó una disminución del porcentaje de humedad del suelo hasta los $40 \mathrm{~cm}$ de profundidad y a partir de los $60 \mathrm{~cm}$ en adelante se recuperó una mayor disponibilidad de agua nuevamente, (Figura 4). 

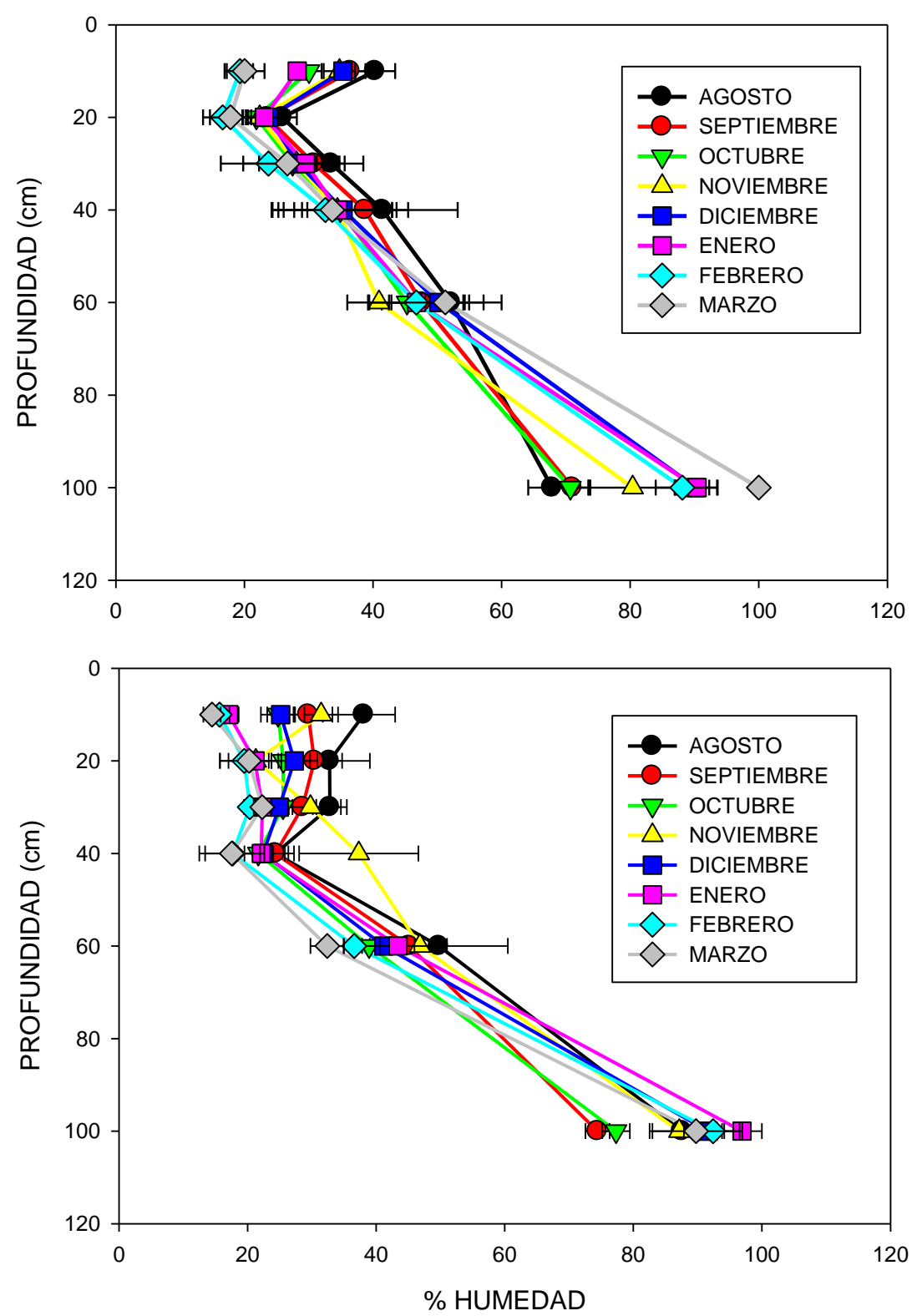

Figura 4. Variación mensual de la humedad del suelo de las microcuencas. Superior: Bosque siempreverde; Inferior: Bosque deciduo

El Anova de Kruskall Wallis que comparó los índices de escorrentía para los dos bosques nativos dio como resultado que no hay diferencias significativas $\left(x^{2}=8,000 ; H=5,338 ; g l=7 ; P=0,618\right)$. Así mismo con este análisis se comparó los caudales para los dos bosques nativos, y tampoco se encontraron diferencias significativas $\left(x^{2}=4,000 ; H=4,279 ; g l=7 ; P=0,747\right)$.

\section{Discusión}

Las interacciones entre la vegetación y los componentes del balance hídrico son de significancia considerable desde el punto de vista fisiológico, ecológico e hidrológico. En las microcuencas del presente estudio, los valores de precipitación registrados fueron mayores en el bosque deciduo, sin embargo la cantidad de agua de escorrentía resultó ser menor que la del bosque siempreverde, porque los procesos hidrológicos que transfieren las precipitaciones netas a los 
cursos de agua y que además recargan las aguas subterráneas, varían de acuerdo a la topografía, el suelo, las características hidrogeológicas y características de las lluvias (Weatherhead y Howden, 2009). Komatsu et al. (2010) manifiestan que una cuenca cubierta con bosques de coníferas siempreverde presenta valores más bajos de escorrentía superficial y mayor pérdida por evapotranspiración que una cuenca con bosques deciduos en regiones con altas precipitaciones de invierno. En nuestro caso, el sitio de Bonifacio que tiene presencia de bosque siempreverde, presentó valores más altos de escorrentía que la cuenca con bosque deciduo. Esto se puede explicar por las menores tasas de transpiración de las especies arbóreas siempreverde y asociadas a un menor aumento de la biomasa foliar durante el período primavera-verano.

La humedad del suelo mostró diferencias para los dos sitios, en el bosque siempreverde, se notó claramente un mayor consumo de agua en los primeros $20 \mathrm{~cm}$ del perfil de suelo, y después a los $30 \mathrm{~cm}$ de profundidad nuevamente aumentó el contenido de humedad aún en los meses con poca precipitación. En los $100 \mathrm{~cm}$ de profundidad el contenido de agua del suelo osciló entre el 70 y $90 \%$, este comportamiento podría deberse a un cambio en el nivel de profundidad edáfica y con los diferentes periodos de lluvia. El menor contenido de humedad del suelo en las capas superficiales puede ser un indicio de la presencia de flujos inestables causados por suelos hidrofóbicos, en suelos derivados de ceniza volcánica (Gonzaga, 2002). Además las especies del bosque siempreverde generalmente presentan un crecimiento radicular lateral que sobrepasa la proyección del dosel, es decir, tienen una baja exploración en profundidad (Gonzaga, 2002). La captación de agua del suelo por las plantas, está fuertemente determinada por el control fisiológico de pérdida de agua, a través de una combinación de fluctuaciones en la conductancia estomática y la cantidad de follaje (Roberts, 2000).

En el bosque deciduo, el mayor consumo de agua fue registrado a los $40 \mathrm{~cm}$ de profundidad, donde el porcentaje de humedad promedio se encontró entre el 20 y $30 \%$ a pesar de la diferencia estacional. En los $100 \mathrm{~cm}$ de profundidad el valor del contenido de agua del suelo llegó incluso a un valor cercano al $100 \%$. Esta respuesta podría deberse al tipo del suelo, de acuerdo al estudio realizado por Reyes, Gerding, y Donoso (2007), quien trabajó en el mismo sitio de la presente investigación, mostró que el suelo presentó buenas condiciones de fertilidad, reflejado principalmente en el mayor espacio arraigable y mejores regímenes de agua (mayor capacidad de agua aprovechable), de aire (drenaje interno moderado) y de elementos.

La transpiración de la mayoría de las especies vegetales está determinada por la demanda climática, de mecanismos fisiológicos relacionados con la respuesta estomática a factores ambientales, del índice de área foliar y disponibilidad de agua en el suelo (Hernández, 2001). Las especies del bosque siempreverde no presentaron un patrón marcado en la variación estacional de la transpiración, ya que después de los meses de invierno incrementaron sus valores hasta el mes de octubre y cuando empezó la primavera descendió nuevamente este índice. Este 
comportamiento podría indicar mecanismos eficientes de control estomático de las pérdidas de agua como respuesta a los cambios en las condiciones ambientales (Gonzaga, 2002).

En el bosque deciduo, en los meses de invierno, la transpiración fue mucho menor que en el bosque siempreverde, la reducción del área foliar, influye en las pérdidas por intercepción de las lluvias y en la transpiración (Roberts, 2000). Pero a partir del mes de octubre la respuesta frente a los cambios climáticos y fisiológicos propios de estas especies indujeron a incrementar esta variable, cuyo comportamiento pudo provocar la disminución de la escorrentía.

El índice de área foliar, presentó un incremento mayor en el bosque deciduo a partir de la primavera que es cuando empieza su máximo desarrollo, hasta alcanzar un valor superior a 6 al final del verano. Estos valores son similares con los presentados por Roberts (2000) donde menciona un LAI entre 5 y 6 en este tipo de bosques. En cambio, en el bosque siempreverde, que presentó un incremento paulatino del área foliar total del bosque, llegó solamente hasta un valor levemente superior a 4. Las correlaciones mostraron que solo en el bosque deciduo existió correlación significativa entre el crecimiento de la biomasa foliar y la transpiración, mientras que el crecimiento de la biomasa foliar no tiene incidencia en las tasas de transpiración de un bosque siempreverde. El índice de área foliar pudo haber variado ampliamente entre los tipos y especies de plantas en respuesta al agua disponible y el estado nutricional del suelo (Roberts, 2000).

La evapotranspiración mensual resultó ser independiente del índice de área foliar, por lo que este comportamiento estaría más relacionado con la cantidad de agua en el suelo. Las pérdidas de agua que se producen por evapotranspiración constituyen un componente importante en el ciclo del agua, determinadas sobre todo por la energía disponible, la disponibilidad de agua (a través de la humedad del suelo o agua subterránea), el efecto secante del aire, la cubierta vegetal y características de la vegetación. (Guardiola-Claramonte, Troch, Ziegler, Giambelluca, Vogler, y Nullet, 2010).

La intercepción de copas de cada una de las especies arbóreas es un factor importante en las pérdidas de agua de un ecosistema boscoso (Huber e Iroumé 2001). Así, la cantidad de agua que llega al suelo es diferente para cada una de las coberturas. Ya que el bosque deciduo presentó mayor biomasa foliar en la primavera-verano, es posible inferir que la intercepción en esta cuenca también pudo ser mayor al menos en esa etapa estacional y, por lo tanto, la precipitación neta sería menor. De tal manera que se podría estar sobrestimando los valores de entrada en el balance hídrico. Sin embargo, se podría tomar como referencia los valores obtenidos en estudios anteriores de Huber e Iroumé (2001) donde muestran que las pérdidas por intercepción en Nothofagus obliqua es de un 10\%. Por otro lado, Fuentes, Hernández, Caldentey, y Castillo (1994) señalan pérdidas por intercepción entre el 15 y 17\% en renoval de roble. En el caso de bosques siempreverde adultos, Huber y Oyarzún (1992) señalan valores de intercepción cercanos 
al 30\% en un lugar cercano a Valdivia. Indudablemente que estos valores de intercepción, que no se midieron en este trabajo, podrían afectar de alguna manera los cálculos del balance hídrico.

\section{Conclusiones}

El bosque deciduo durante el período de transición primavera-verano mostró un cambio en sus tasas de transpiración, viéndose reflejada en la menor cantidad de agua de escorrentía de salida en la cuenca, en comparación con el bosque siempreverde.

Las tasas de transpiración de las especies del bosque deciduo estuvieron significativamente correlacionadas con el incremento de la biomasa foliar, en comparación con las especies del bosque siempreverde que no tuvieron una relación significativa.

La disminución del contenido de agua del suelo fue mayor en el bosque deciduo que en el bosque siempreverde. Esto indicaría que los árboles del bosque deciduo extraen agua a mayores profundidades que los del bosque siempreverde.

El balance hídrico indicó que la evapotranspiración total es mayor en el bosque deciduo que en el bosque siempreverde.

La escorrentía total fue menor en el bosque deciduo que en el bosque siempreverde, aunque no se encontraron diferencias significativas entre los dos bosques. Esto sugiere que las especies arbóreas del bosque siempreverde son más conservativas con respecto al uso del agua, que las especies deciduas.

\section{Bibliografía}

Barrantes, O., Gracia, C.A. (1989) Estimación del área foliar a través de la superficie de albura en Pinus sylvestris. CIHEAM - Options Mediterraneennes, Serie Seminarios 3: 53 - 56.

Cornish, P.M., Vertessy, R.A. (2001) Forest age-induced changes in evapotranspiration and water yield in a eucalypt forest. Journal of Hydrology 242: $43-63$.

Echeverría, C., Huber, A., Taberlet, F. (2007) Estudio comparativo de los componentes del balance hídrico en un bosque nativo y una pradera en el sur de Chile. Bosque 28(3): 271-280 
Flores, F., Lee Allen, H. (2004) Efectos del clima y capacidad de almacenamiento de agua del suelo en la productividad de rodales de pino radiata en Chile: un análisis utilizando el modelo 3-PG. Bosque 25(3): 11-24

Fuentes, J., Hernández, J., Caldentey, J., Castillo, H. (1994) Efectos sobre la redistribución de las precipitaciones y los contenidos de humedad del suelo por la transformación de un bosque de Nothofagus obliqua a una plantación de Pinus radiata en la cordillera de Nahuelbuta. Ciencias Forestales 9 (1-2): 23-39

Gonzaga, L. (2002) Hidrología Forestal Influencia de los bosques. Universidad Nacional de Colombia - Medellín. 227pp.

Guardiola-Claramonte, M., Troch, P., Ziegler, A., Giambelluca, M., Vogler, J. Nullet, M. (2010) Hydrologic effects of the expansion of rubber (Hevea brasiliensis) in a tropical catchment. Ecohydrology DOI: 10.1002/ eco.110

Hernández, R. (2001) Balance Hídrico de la planta. Universidad de los Andes, Mérida Venezuela

Huber, A., Oyarzún, C., M.M. Oñate (1986) Factores reguladores de la transpiración potencial de algunas especies arbóreas del bosque siempre verde del sur de Chile. Turrialba 36 (3): 329 336.

Huber A, Oyarzún C. (1992) Redistribución de las precipitaciones en un bosque siempreverde del sur de Chile. Turrialba 42: 192-199.

Huber, A., Iroume, A. (2001) Variability of annual rainfall partitioning for different sites and forest covers in Chile. Journal of Hydrology 248:78-92

Iroume, A., Huber, A. (2000). Intercepción de las lluvias por la cubierta de bosques y efecto en los caudales de crecida en una cuenca experimental en Malalcahuello, IX Región, Chile. Bosque 21(1): 45-56.

Komatsu, H., Kume, T., Otsuki, K. (2010) Increasing annual runoff - broadleaf or coniferous forests?.Hydrological Processes (wileyonlinelibrary.com). DOI: 10.1002/ hyp.7898

Krämer, I., Hölscher, D. (2010) Soil water dynamics along a tree diversity gradient in a deciduous forest in Central Germany. Ecohydrology DOI: 10.1002/ eco.103.

Lara, A., Rutherford, P., Montory, C. (1999). Vegetación de la Eco- Región de los bosques Valdivianos. Proyecto Binacional Chile y Argentina. Boletín técnico FVSA, No. 51 
Lara A, Little C, Urrutia R, McPhee J, Alvarez C, Oyarzun C, Soto D, Nahuelhual L, Donoso P, Pino M. (2009). Ecosystem services as an opportunity for the conservation and management of native forests in Chile. Forest Ecology and Management 258: 415-424.

Mendoza, M., Bocco, G., López, E., Bravo, M. (2002) Implicaciones hidrológicas del cambio de la cobertura vegetal y uso del suelo: una propuesta de análisis espacial a nivel regional en la cuenca cerrada del lago de Cuitzeo, Michoacán. Investigaciones Geográficas 49: 92-117.

Oyarzún, CE., Huber, A., Vásquez, S. (1984) Balance hídrico en tres plantaciones de Pinus radiata. Bosque $6(1): 3-14$

Oyarzún, CE., Nahuelhual,L., Núñez, D. (2006) Los servicios ecosistémicos del bosque templado lluvioso: producción de agua y su valoración económica. Revista Ambiente y Desarrollo 20(3) $-21(1): 88-95$.

Oyarzún, CE., Zuñiga, A. (2009) Water Yield, Seasonal Growth Vegetation and Transpiration rate of small headwater catchments in southern Chile Proyecto Fondecyt 1090345.

Oyarzún, CE., Verhoest N, Staelens J, Godoy R. (2009) Effects of management practices on water yield in small headwater catchments at Cordillera de los Andes in southern Chile. In: N. Verhoest, P. Boeckx, C. Oyarzún \& R. Godoy (eds) Ecological Advances on Chilean Temperate Rainforests. Academia Press, Belgium. P. 55-63.

Oyarzún, CE., Godoy R., Staelens J., Donoso P., Verhoest NEC. (2011). Seasonal and annual throughfall and stemflow in Andean temperate rainforests. Hydrological Processes 25: 623633.

Pizarro, R., Vallejos, O., Balocchi, F., Morales, C., León, L. (2005) Determinación de las intercepciones de agua en bosques nativo maulino y en plantaciones de Pinus radiata D. Facultad de Ciencias Forestales. Universidad de Talca. Talca - Chile.

Quintana, C. (2009) Estimación de evapotranspiración utilizando datos de sensores remotos satelitales. Universidad de Concepción Departamento de Recursos Hídricos. Chile.

Reyes, R., Gerding, V., Donoso, C. (2007) Crecimiento de una plantación de Nothofagus nervosa durante veinte años en Valdivia Bosque 28 (2): 129 - 138

Roberts, J. (2000) The influence of physical and physiological characteristics of vegetation on their hydrological response. Hydrological Processes 14: $2885-2901$

Schelde, K. (1996) Modeling the forest energy and water balance. Department of Hydrodynamics and Water Resources. Technical University of Denmark. 
Vazken, A. (2003) Waters and forests: from historical controversy to scientific debate. Journal of Hydrology 291:1-27.

Weatherhead, E.K., Howden,N.J.K. (2009) The relationship between land use and surface water resources in the UK. Elsevier 26S S243 - S250

Whitehead, D., Kelliher, F., Frampton,C., Godfrey, M. (1994) Seasonal development of leaf area in a young, widely spaced Pinus radiata D. Don stand. Tree Physiology14:1019- 1038.

Yrarrázabal, P., Antoñanzas, I. (2008). Árboles nativos de Chile. Fundación Huinay. Chile 